\title{
Immunoassay of platelet-derived growth factor in the blood of patients with diabetes mellitus
}

\author{
A.A.Harrison ${ }^{1}$, P. R.Dunbar ${ }^{2}$, T.J. Neale ${ }^{2}$ \\ ${ }^{1}$ Wellington Regional Rheumatology Unit, Hutt Hospital, Lower Hutt, New Zealand \\ ${ }^{2}$ Department of Medicine, Wellington School of Medicine, Wellington, New Zealand
}

\begin{abstract}
Summary Platelet-derived growth factor (PDGF) is a powerful mitogen for many cell types, and is believed to play a major role in wound healing when released from platelets at sites of injury. In diabetes mellitus, it has been proposed that premature release of PDGF from platelets impairs the ability of platelets to initiate healing, and also accelerates the development of diabetic complications such as angiopathy by increasing plasma-borne PDGF. However, plasma samples from diabetic patients have not previously been assayed for PDGF using suitable techniques. A sensitive monoclonal enzyme-linked immunoassay for PDGF was applied to plasma and serum samples from 18 healthy control subjects and 60 diabetic patients. Neither plasma nor serum PDGF concentrations differed significantly between control subjects, insulin-dependent, and non-insulin-dependent diabetic patients. However, $23 \%$ of the diabetic subjects had serum PDGF levels above the control range. Limited joint mobility, which is characterised
\end{abstract}

by joint contractures and collagen deposition in the skin, and is associated with microvascular disease, was used as a marker of diabetic complications. Limited joint mobility affected $43 \%$ of the diabetic subjects. Patients with moderate limited joint mobility had had diabetes significantly longer than those without limited joint mobility (means 17 years and 9 years, respectively, $p=0.008$ ). However, limited joint mobility was not associated with elevated serum or plasma PDGF in insulin-dependent or non-insulin-dependent diabetes. We conclude that complications of diabetes are unlikely to be caused by changes in systemic levels of PDGF. The delayed healing associated with diabetes is not due to a deficit in PDGF available from platelets. [Diabetologia (1994) 37: 1142-1146]

Key words Platelet-derived growth factor, limited joint mobility, diabetes mellitus.
Platelet-derived growth factor (PDGF) is a powerful stimulator of fibroblast mitogenesis and fibroproliferative responses [1]. At sites of platelet adherence,

Received: 23February 1994

and in revised form: 22 June 1994

Corresponding author: Professor T.J. Neale, Department of Medicine, Wellington School of Medicine, P. O. Box 7343, Wellington South, New Zealand

Abbreviations: PDGF, Platelet-derived growth factor; PF4, platelet factor 4; TGF- $\beta$, transforming growth factor-beta; LJM, limited joint mobility; IDDM, insulin-dependent diabetes mellitus; NIDDM, non-insulin-dependent diabetes mellitus.
PDGF liberated from platelets during the release reaction is believed to play a major role in wound healing. In diabetes mellitus, abnormalities in platelet function have frequently been observed, but while enhanced platelet aggregation responses have been a consistent finding, reports of altered release of platelet constituents have been more controversial [2]. Some previous work has shown that the platelets of diabetic patients contain less fibroblast mitogenic activity than platelets from control subjects, and it has been proposed that platelets from patients with diabetes release PDGF prematurely $[3,4]$. The resulting platelet PDGF deficit might contribute to the delayed healing seen in diabetes [3], while increased plasma PDGF concentrations could be involved in 
Table 1. Subjects studied

\begin{tabular}{llll}
\hline & \multicolumn{2}{l}{ Diabetic patients } & $\begin{array}{c}\text { Control } \\
\text { subjects }\end{array}$ \\
\cline { 2 - 3 } & IDDM & NIDDM & \\
\hline$n$ & 18 & 42 & 18 \\
Mean age (years) & 37 & $56^{\mathrm{a}}$ & 42 \\
Age range (years) & $17-75$ & $20-71$ & $14-70$ \\
Treatment & Insulin \& diet & $\begin{array}{l}\text { Diet/oral } \\
\text { hypogly- }\end{array}$ & - \\
caemic & \\
drugs & \\
Mean diabetes & 14 & 10 & - \\
duration (years) & & & \\
\hline
\end{tabular}

a Subjects with NIDDM were significantly older than the other two groups, $p<.001$

Table 2. Criteria for LJM (after Rosenbloom et al. [8])

No LJM normal, ${ }^{a}$ equivocal or unilateral findings

Mild LJM one or two interphalangeal joints or one large joint or only metacarpophalangeal joints bilaterally

Moderate LJM three or more interphalangeal joints or one finger joint and one large joint bilaterally

Severe LJM moderate limitation combined with cervical spine involvement or hand deformity at rest

a Where normal ranges of movement are defined as greater than or equal to:

$180^{\circ}$ extension at the proximal interphalangeal joints

$60^{\circ}$ extension at metacarpophalangeal joints

$70^{\circ}$ extension at the wrist

$180^{\circ}$ extension at the elbow

$100^{\circ}$ flexion at the ankle

$35^{\circ}$ lateral flexion at the cervical and thoracolumbar spine

other complications of diabetes such as angiopathy [4]. However, plasma PDGF concentrations have not been measured in diabetic patients with a sensitive specific assay $[4,5]$. In the current study a sensitive ELISA has been used to assess PDGF concentrations in the plasma and serum in patients with both insulin-dependent diabetes (IDDM) and non-insulin dependent diabetes (NIDDM).

In addition we assessed limited joint mobility (LJM) in all diabetic subjects, as a relevant clinical correlate of diabetic complications with a potential relationship to PDGF action. LJM in diabetes was first described in 1957 [6], and most descriptive studies have since reported its prevalence as between $30 \%$ and $40 \%$ [7-9]. In children and adults with either IDDM or NIDDM, LJM is associated with thickening of the joint capsule and skin, sclerodactyly, restrictive pulmonary disease and microvascular complications, particularly retinopathy, perhaps suggest- ing a common pathogenesis [7]. Histologically, the sclerodactyly of LJM resembles scleroderma, with loss of dermal appendages and increased deposition of collagen in the dermis [10]. Since LJM is associated with increased fibrosis and collagen deposition, as well as with microvascular disease, it provides an ideal syndrome for the evaluation of any potential correlation between fibrotic diabetic complications and circulating PDGF concentrations. To date there has been no evaluation of blood PDGF concentrations in LJM.

\section{Subjects, materials and methods}

Subjects. The subjects studied are described in Table 1. Diabetic subjects were adult patients attending the diabetes clinic consecutively at Hutt Hospital over a 3-month period. Informed consent was obtained and details of type and duration of diabetes were recorded. Subjects were examined and classified for presence and severity of LJM using the criteria described by Rosenbloom et al. [8] shown in Table 2. A blood sample was taken for estimation of platelet count by Coulter counter. Samples of serum and platelet-free plasma were then obtained, using a previously-validated method which minimises platelet degranulation during plasma collection [11]. Plasma and serum samples from 18 healthy subjects were used as controls. The study design had the approval of the Research Ethical Committee of the Wellington Area Health Board.

PDGF ELISA. All samples were assayed for PDGF as previously described [11], by the same worker on the same day. Briefly, microtitre plates were coated with PGF-007, an IgG1 monoclonal antibody with specificity for PDGF B-chain (Mochida Pharmaceutical, Tokyo, Japan) [12]. Duplicate samples or standard dilutions of PDGF (PDGF Inc., Boston, Mass., USA) were then applied, followed by goat anti-human PDGF (Collaborative Research, Boston, Mass., USA), rabbit antigoat $\operatorname{IgG}$, goat peroxidase-anti-peroxidase complex (Dako, Glostrup, Denmark), and O-phenylenediamine as substrate. Absorbances at $492 \mathrm{~nm}$ were interpreted using MacRIA, an immunoassay standard curve interpolation computer software program [13]. The detection limit of this assay is $125 \mathrm{pg} / \mathrm{ml}$, and the coefficients of variation for serum are less than $5 \%$ within an assay, and approximately $8 \%$ between assays [11]. A measure of total platelet PDGF load per ml of blood was obtained by subtracting the PDGF concentration found in each individual's plasma from that found in serum. Previous work has demonstrated that PDGF concentrations obtained from platelet lysates correlate with serum PDGF concentrations [5].

PF4 immunoassay. PDGF is released by degranulating platelets, and plasma levels of PDGF can be measured reliably only when platelet degranulation is minimised [11]. Since platelet alpha granules contain both PDGF and PF4, PF4 concentrations were measured in each plasma preparation using a commercial immunoassay (Behring, Marburg, Germany), to assess whether significant platelet degranulation had occurred.

\section{Statistical analysis}

Normally distributed variables such as age, length of diabetes history and platelet count were compared using Student's $t$ test. Plasma PDGF, serum PDGF and plasma PF4 concentrations were compared between diabetic and control groups us- 


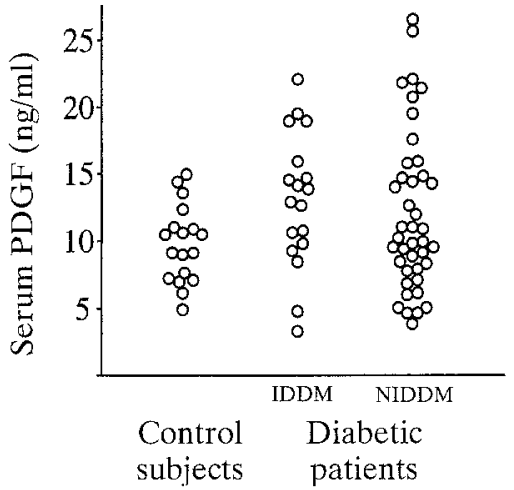

Fig. 1. Serum PDGF in normal control subjects and diabetic patients

ing the non-parametric Mann-Whitney $U$ test. Diabetic patients were divided into IDDM and NIDDM groups, and results compared simultaneously with those from the control group using the non-parametric multi-group Kruskal-Wallis test. Diabetic patients were sub-divided according to whether their serum PDGF values fell within the control range, and LJM scores were compared using the Mann-Whitney U test. Correlation between plasma and serum PDGF values and LJM score was also sought using $X-Y$ correlation matching. Spearman rank correlation was used to express bivariate relationships. $P$-values greater than 0.05 were not considered statistically significant, and are not specified in the text.

\section{Results}

Plasma samples from 55 of 60 diabetic subjects had $\mathrm{PF} 4$ concentrations between 1.9 and $6.4 \mathrm{ng} / \mathrm{ml}$ (manufacturer's normal range 1.4-6.1 ng/ml). Four samples had PF4 concentrations between 14.9 and $22.0 \mathrm{ng} / \mathrm{ml}$, but this moderate elevation was unlikely to be due to ex vivo platelet degranulation since PDGF was undetectable. One diabetic patient had a plasma PF4 concentration of $198 \mathrm{ng} / \mathrm{ml}$ and a plasma PDGF concentration of $660 \mathrm{pg} / \mathrm{ml}$, and was excluded from PDGF analysis. One control sample had a PF4 concentration of $30.2 \mathrm{ng} / \mathrm{ml}$ but did not have detectable PDGF and was therefore included in PDGF analysis.

After exclusion of the diabetic subject with ex vivo degranulation, there was no difference in plasma PF4 concentrations between healthy and diabetic subjects, or amongst the IDDM, NIDDM and control groups. PF4 levels did not correlate with platelet concentrations.

PDGF was detected in the plasma of eight of 59 diabetic patients (range 250 to $4830 \mathrm{pg} / \mathrm{ml}$ ) and three of 18 control subjects (range 250 to $1250 \mathrm{pg}$ / $\mathrm{ml}$ ). Plasma PDGF and plasma PF4 concentrations did not correlate $(r=-0.209)$. There was no statistical difference in plasma PDGF concentrations between diabetic patients and control subjects, or between IDDM and NIDDM patients. Age and dura- tion of diabetes did not correlate with plasma PDGF concentrations.

PDGF was detected in the serum of all 60 diabetic patients (range 3310 to 26540 , median $10980 \mathrm{pg} / \mathrm{ml}$ ) and all 18 healthy control subjects (range 4980 to 15070 , median 9920 pg/ml) (Fig. 1). Serum PDGF concentrations were not statistically different between diabetic patients and control subjects, or amongst IDDM, NIDDM, and control subjects. However, 4 (22\%) IDDM, and 10 (24\%) NIDDM subjects had serum PDGF concentrations above the control range (Fig. 1). Neither age nor duration of diabetes correlated with serum PDGF levels.

Since plasma PDGF concentrations were so low in comparison to those in serum, calculation of PDGF derived from platelets yielded similar results. There was no statistical difference between platelet PDGF per $\mathrm{ml}$ of blood in diabetic subjects (range 3310 to 26540 , median $11050 \mathrm{pg} / \mathrm{ml}$ ) and control subjects (range 4980 to 15070 , median $9920 \mathrm{pg} / \mathrm{ml}$ ), and this lack of significance held when diabetic patients were subdivided as to type.

Of the 60 diabetic patients, $26(43 \%)$ had LJM: 13 had mild and 13 had moderate LJM; none had severe LJM. There was no significant difference in the ages of the LJM groups, but length of diabetic history increased with severity of LJM (mean 9 years for subjects with no LJM vs 17 years for moderate LJM, $p=0.008)$.

There were no correlations between plasma or serum PDGF concentrations and the degree of LJM. Further, when diabetic patients with elevated serum PDGF concentrations were compared with all other diabetic patients, no significant difference in LJM scores emerged. The lack of association between severity of LJM and PDGF concentrations in the serum and plasma persisted when the IDDM and NIDDM groups were considered separately. Platelet count did not differ with respect to type of diabetes.

\section{Discussion}

PDGF is a powerful mitogen for mesenchymal cells, and is suspected of involvement in a wide range of fibrotic conditions [1]. Platelets from diabetic patients have demonstrated reduced mitogenic activity for fibroblasts, and it has been proposed that this is due to loss of platelet PDGF in to the plasma $[3,4]$. Such release could impair the ability of platelets to initiate healing, and accelerate the development of diabetic complications such as angiopathy by increasing plasma-borne PDGF [3, 4]. Previous assays of PDGF concentrations in diabetic patients have lacked suitability or sensitivity for use in plasma $[4,5]$. An ELI$\mathrm{SA}$ with a detection limit of $2 \mathrm{ng} / \mathrm{ml}$ detected PDGF in the plasma of only one of 28 diabetic patients [5]. 
Our assay was more sensitive, with a detection limit of $125 \mathrm{pg} / \mathrm{ml}$, and allowed measurement of plasma PDGF in 8 of 59 diabetic patients. We found no evidence of an increase in plasma PDGF concentrations in diabetic patients, and no reduction in a derived measurement of platelet PDGF content. We conclude that any reduction in platelet mitogenic activity for fibroblasts is not caused by loss of platelet PDGF into the plasma. Since platelets contain several other potential modulators of fibroblast growth, such as members of the TGF- $\beta$ family, it is possible that altered accumulation or release of other platelet constituents is responsible for the reported changes in mitogenic activity $[4,14]$. It is also of note that at least one previous study found no reduction in the growth-promoting activity of platelets from diabetic patients [5]. Technical difficulties associated with platelet collection, separation, and washing may account for some of the discrepancies amongst the studies to date [3-5].

Previous studies of PDGF in the serum of diabetic patients have yielded conflicting results. Nakashima et al. [5], using an ELISA, found no significant statistical difference between serum PDGF concentrations in 28 NIDDM diabetic subjects and 11 control subjects [5]. Lev-Ran and Hwang [15], using a radioimmunoassay, found a small but statistically significant increase in serum PDGF concentrations in 19 diabetic patients of mixed type compared with 13 control subjects. The accuracy of competitive radioimmunoassay for PDGF in blood may be compromised by the presence of binding proteins in plasma [16], a problem surmounted by non-competitive ELISA's $[5,11]$. We studied a much larger number of diabetic patients than had previous workers, and found elevated serum PDGF concentrations in about $23 \%$ of both IDDM and NIDDM patients. It seems likely, therefore, that a proportion of diabetic patients have elevated serum PDGF concentrations, but that these measurements do not cause a statistically significant difference between groups when larger numbers of diabetic patients are compared with control subjects. Importantly, in our study an increase in serum PDGF concentrations did not correlate with the clinical marker of LJM, and was not related to length of diabetes. Previous work also showed no correlation between an increased serum PDGF concentration and duration of diabetes, mode of therapy, or control of glycaemia, although correlation with clinical complications was not studied [15].

Since the majority of serum PDGF is derived from ex vivo platelet degranulation, these studies suggest that the platelets of diabetic patients contain equivalent or greater amounts of PDGF than the platelets of control subjects. However, diabetic serum has reduced growth-promoting activity [5], as might be expected from data demonstrating a similar mitogenic deficit in diabetic platelets. Clearly, this observation is not due to a lack of platelet-derived PDGF in serum. As has previously been postulated, the presence of growth-inhibitory factors, or the lack of other growth-stimulatory factors, may be responsible for any reduction in the mitogenic capacity of diabetic serum $[5,14]$. Glucose itself may have an inhibitory effect in some cell proliferation assays [17]. It is also possible that non-enzymatic glycation of growth-promoting cytokines such as PDGF may reduce their biological activity, despite demonstration of normal concentrations of immunoreactive molecules.

The association of LJM with microvascular complications, independent of age and duration of diabetes [9], afforded the ideal clinical marker with which to compare PDGF concentrations. The lack of any correlation between LJM and either plasma or serum PDGF concentrations suggests that microvascular complications of diabetes are unlikely to be influenced by systemic PDGF concentrations. Serum factors with different physical properties from PDGF have previously been implicated in diabetic retinopathy [18].

The prevalence of LJM in diabetes in our investigation ( $43 \%$ ) was similar to that found in other studies. Clinically and histologically, the sclerodactyly of LJM resembles scleroderma, a disease in which we were also unable to demonstrate elevation in plasma PDGF concentrations compared with normal and disease control subjects [11]. A previous bioassay study had shown increased plasma growth promoting activity on fibroblasts [19], and an immunohistological study had demonstrated PDGF in scleroderma skin biopsies [20]. It is possible that immunohistology would yield similar results in LJM, supporting a local rather than a systemic role for PDGF in this condition.

In conclusion, we have measured PDGF concentrations in the plasma, serum and platelets of patients with IDDM and NIDDM, and sought correlations between changes in PDGF concentrations and the clinical syndrome of LJM. Although $23 \%$ of patients with both IDDM and NIDDM had serum and platelet PDGF concentrations above the control range, there was no statistical difference between the groups. There was no association between plasma or serum PDGF concentrations and LJM. Previously reported reductions in the growth-promoting activity of platelets and serum from diabetic patients clearly do not relate to changes in PDGF concentrations. This finding does not preclude a local role for PDGF in diabetic vascular disease or LJM.

Acknowledgements. We are grateful to Drs. D.Carroll and $\mathrm{R}$. Toomath and the staff of Hutt Hospital Diabetes Clinic, in particular Mrs. P.Easter, for their help in enrolling subjects in this study. Aspects of this work were supported by the Health Research Council of New Zealand, and the Diabetes Research Trust Fund of the Wellington Area Health Board. 


\section{References}

1. Ross R (1989) Platelet-derived growth factor. Lancet I: 1179-1182

2. Winocour PD (1992) Platelet abnormalities in diabetes mellitus. Diabetes 41: 26-31

3. Caenazzo A, Pietrogrande F, Polato G, Piva E, Sartori D, Girolami A (1991) Decreased platelet mitogenic activity in patients with diabetes mellitus. Haematologia 24: 241247

4. Guillausseau PJ, Dupuy E, Bryckaert MC et al. (1989) Platelet-derived growth factor (PDGF) in type 1 diabetes mellitus. Eur J Clin Invest 19: 172-175

5. Nakashima N, Umeda F, Yamauchi Tet al. (1992) Plateletderived growth factor and growth-promoting activity in the serum samples and platelets of patients with non-insulin-dependent diabetes mellitus. J Lab Clin Med 120: 78-85

6. Lundbaek K (1957) Stiff hands in long-term diabetes. Acta Med Scand 158: 447-451

7. Kapoor A, Sibbitt WL (1989) Contractures in diabetes mellitus: the syndrome of limited joint mobility. Semin Arthritis Rheum 18: 168-180

8. Rosenbloom AL, Silverstein JH, Lezotte DC, Richardson K, McCallum M (1981) Limited joint mobility in childhood diabetes mellitus indicates increased risk for microvascular disease. N Engl J Med 305: 191-194

9. Jennings AM, Milner PC, Ward JD (1989) Hand abnormalities are associated with the complications of diabetes in type 2 diabetics. Diabetic Med 6: 43-47

10. Buckingham BA, Uitto J, Sandborg C et al. (1984) Scleroderma-like changes in insulin-dependent diabetes mellitus: clinical biochemical studies. Diabetes Care 7: 163-169

11. Dunbar PR, Harrison AA, Corkill MM, Neale TJ (1993) Immunoassay of platelet-derived growth factor in the plas- ma of patients with scleroderma. Br J Rheumatol 32: 966971

12. Shiraishi T, Morimoto S, Itoh K et al. (1989) Radioimmunoassay of human platelet-derived growth factor using monoclonal antibody toward a synthetic 73-97 fragment of its B-chain. Clin Chim Acta 184: 65-74

13. McIntosh J, Murray-McIntosh R (1991) MacRIA 1.01. Wellington School of Medicine, Wellington, N.Z.

14. Koschinsky T, Bunting CE, Rütter R, Gries FA (1983) Vascular growth factors and the development of macrovascular disease in diabetes mellitus. Horm Metab Res [Suppl] 15: 23-27

15. Lev-Ran A, Hwang DL (1990) Epidermal growth factor and platelet-derived growth factor in blood in diabetes mellitus. Acta Endocrinol (Copenh) 123: 326-330

16. Huang JS, Huang SS, Deuel TF (1983) Human platelet-derived growth factor: radioimmunoassay and discovery of a specific plasma-binding protein. J Cell Biol 97: 383-388

17. Umeda F, Yamauchi T, Nakashima N et al. (1991) Glucose reduces PDGF production and cell proliferation of cultured vascular endothelial cells. Horm Metabol Res 23: 274-277

18. Petty RG, Pearson JD, Morgan DML, Mahler RF (1988) Stimulation of endothelial cell growth by sera from diabetic patients with retinopathy. Lancet I: 208-211

19. Pandolfi A, Florita M, Altomare G, Pigatto P, Donati MB (1989) Increased plasma levels of platelet-derived growth factor activity in patients with progressive systemic sclerosis. Proc Soc Exp Biol Med 191: 1-4

20. Gay S, Jones REJ, Huang GQ, Gay RE (1989) Immunohistologic demonstration of platelet-derived growth factor (PDGF) and sis-oncogene expression in scleroderma. J Invest Dermatol 92: 301-303 